\title{
Sporadic giant intra-abdominal desmoid tumor: A radiological case report
}

\author{
KARLA KOVAČEVIĆ ${ }^{1 *}$, DRAGICA OBAD-KOVAČEVIĆ ${ }^{2}$ and JELENA POPIĆ-RAMAČ ${ }^{1,2^{*}}$ \\ ${ }^{1}$ School of Medicine, University of Zagreb; ${ }^{2}$ Department of Diagnostic and Intervention \\ Radiology, University Hospital Merkur, 10000 Zagreb, Croatia
}

Received January 23, 2017; Accepted February 28, 2017

DOI: $10.3892 / \operatorname{mco} .2017 .1250$

\begin{abstract}
Desmoid tumor (DT) is a locally invasive form of fibromatosis, comprising only $0.03 \%$ of all tumors. DTs occur more frequently in patients with familial adenomatous polyposis and Gardner's syndrome, as intra-abdominal or anterior abdominal wall tumors, whereas sporadic DTs are more likely to be extra-abdominal (only $5 \%$ of sporadic DTs are intra-abdominal). There is also an association of DTs with prior trauma, surgery, estrogen exposure and childbirth. Imaging studies, such as computed tomography (CT) and magnetic resonance imaging (MRI) are used for preoperative diagnosis and for the planning of the surgery. Following surgery, CT and MRI are used to detect recurrence and to monitor the tumor's response to radiotherapy or medical therapy for unresectable or recurrent tumors. We herein report a rare case of a sporadic giant intra-abdominal DT in a 28 -year-old female patient without any predisposing factors, and highlight the importance of including DT in the differential diagnosis of huge intra-abdominal masses.
\end{abstract}

\section{Introduction}

Desmoid tumor (DT), a locally invasive form of fibromatosis, comprises only $0.03 \%$ of all tumors (1). DT may develop in the extremities, chest wall, abdominal wall and intra-abdominally (2). DTs are benign tumors exhibiting infiltrative growth and a tendency for recurrence $(1,3)$. Intra-abdominal DTs are rare and seldom reported in the literature. There is a frequent association of DTs with familiar adenomatous polyposis (FAP) and Gardner's syndrome (1). In patients with FAP and Gardner's syndrome, the tumors are more likely to be intra-abdominal

Correspondence to: Dr Dragica Obad-Kovačević, Department of Diagnostic and Intervention Radiology, University Hospital Merkur, Zajčeva 19, 10000 Zagreb, Croatia

E-mail: dragica.obad. kovacevic@gmail.com

*Contributed equally

Key words: abdomen, oncology, benign tumor, desmoid tumor, computed tomography or located in the anterior abdominal wall (4). By contrast, sporadic DTs are more likely to be extra-abdominal (4), with only $5 \%$ of sporadic DTs located intra-abdominally (5). Other possible triggers for the development of intra-abdominal DTs mentioned in the literature include female gender, childbirth, prior trauma or surgery and estrogen exposure $(1,6,7)$.

\section{Case report}

A 28-year-old woman, without any previous systemic disease, was referred to the Department of Diagnostic and Intervention Radiology, University Hospital Merkur (Zagreb, Croatia) in November, 2016, for abdominal computed tomography (CT) due to occasional abdominal pain of varying intensity for 6 months. There were no other reported symptoms. The patient had no past history of colonic polyps, abdominal trauma or surgical therapy. The family history was negative for FAP. The general physical examination was unremarkable. The analysed blood parameters were within the normal ranges and the tumor markers were negative.

Ultrasound (US) examination revealed a large solid-cystic heterogeneous hypoechoic mass in the upper left abdomen. A contrast-enhanced CT of the abdomen revealed a large heterogeneous solid-cystic intraperitoneal mass (18x14x11 cm) occupying most of the left upper abdomen, extending from the left hemidiaphragm to the upper pole of the left kidney, with partially well-defined margins. The left adrenal gland and left kidney were displaced downward. The mass was inseparable from the adjacent greater curvature of the stomach, left hemidiaphragm, transverse colon, pancreatic tail and spleen. Hyperdense areas of fresh hemorrhage were observed within the tumor tissue (Figs. 1-3). Hypointense infarcted areas were also identified in the spleen.

A subsequent laparotomy revealed a giant tumor located in the left upper abdomen fixed to the greater curvature of the stomach, pancreas, transverse colon, left adrenal gland, left part of the diaphragm and spleen. An en bloc resection of the tumor with the adherent spleen and left adrenal gland, with partial resection of the stomach, pancreas, left hemidiaphragm and transverse colon was performed. The patient tolerated the procedure well and was discharged on the 7 th postoperative day.

Histopathological examination of the mass confirmed the diagnosis of solid-cystic DT, with negative surgical margins 


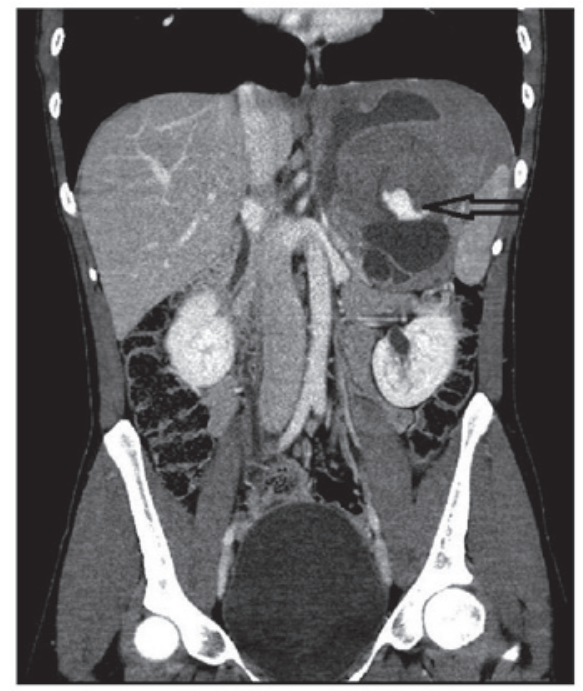

Figure 1. Coronal contrast-enhanced computed tomography image showing a sizeable solid-cystic mass occupying the left upper abdominal quadrant, with an area of hemorrhage (arrow), and displacement of the spleen, pancreas and left kidney.



Figure 2. Coronal contrast-enhanced computed tomography image showing a large heterogeneous mass in the left upper abdomen, with displacement of the stomach and large bowel. The tumor margins are well-defined, apart from the adjacent greater curvature of the stomach.

and hemorrhagic areas. Immunohistochemical analysis revealed that the tumor cells were positive for $\beta$-catenin. Follow-up details are not yet available.

\section{Discussion}

DT is a rare benign tumor with a high propensity for infiltrative growth and local invasion, which tends to recur following local excision, but does not metastasize (1-6). Women are more commonly affected compared with men, with a

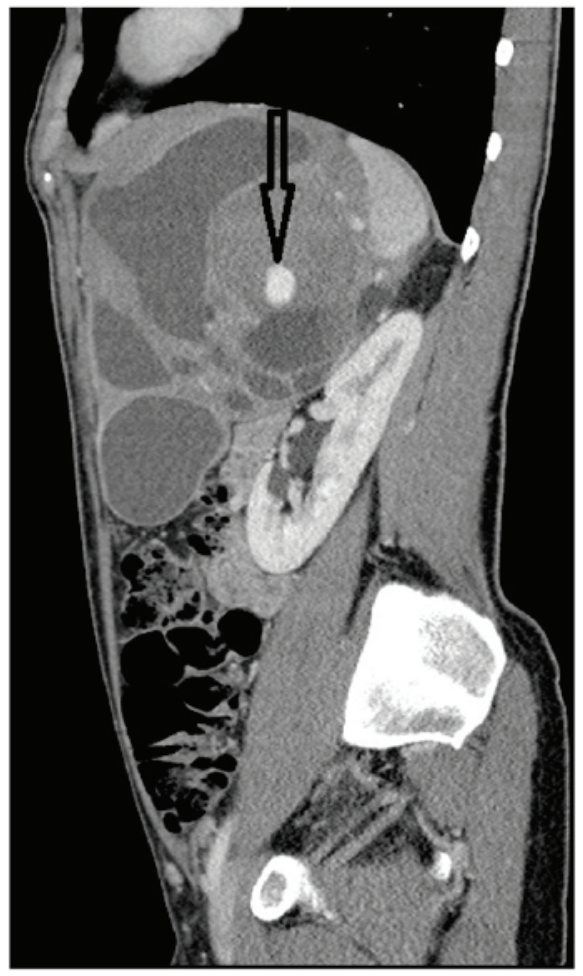

Figure 3. Sagittal contrast-enhanced computed tomography image showing a sizeable expansive lesion in the left upper abdominal quadrant, with an area of hemorrhage (arrow), inseparable from the left hemidiaphragm, with downward displacement of the left kidney.

female:male ratio of 3:1 (8). Two-thirds of the patients are aged 20-40 years (8). Associated conditions include FAP, Gardner's syndrome, trauma, hormonal imbalance and prior surgery (8). DTs are usually solitary, but in $10-15 \%$ of the cases they are multiple (8). Intra-abdominal DTs are able to grow to a large size prior to becoming symptomatic (9).

Imaging studies, such as CT and magnetic resonance imaging (MRI), are used for preoperative diagnosis and for the planning of the surgery. Following surgery, CT and MRI are used for detecting recurrence and to monitor tumor response to radiotherapy or medical therapy for unresectable or recurrent tumors $(4,9)$. The CT and MRI characteristics of intra-abdominal DTs are associated with their histological characteristics and vascularity (9). The typical appearance of an intra-abdominal DT on CT is that of a well-circumscribed solid soft tissue mass, without calcifications (9). Although intra-abdominal DTs may appear to be mostly well-circumscribed on CT and MRI, they are often infiltrative (10). The US characteristics of intra-abdominal DTs are often non-specific (10).

Complete surgical resection with negative pathological margins is the first line of management (10), and careful follow-up is recommended due to the high rate of recurrence (11). Colonoscopy and examination of the eye to exclude Gardner's syndrome are also recommended (11).

In conclusion, we herein presented a case of intra-abdominal DT in a female patient who was negative for the most well-known predisposing factors. The aim of this study was to highlight the importance of including DT in the differential diagnosis of a huge intra-abdominal mass. 


\section{References}

1. Palladino E, Nsenda J, Siboni R and Lechner C: A giant mesenteric desmoid tumor revealed by acute pulmonary embolism due to compression of the inferior vena cava. Am J Case Rep 15: 374-377, 2014

2. Kasper B, Ströbel P and Hohenberger P: Desmoid tumors: Clinical features and treatment options for advanced disease Oncologist 16: 682-693, 2011.

3. Kreuzberg B, Koudelova J, Ferda J, Treska V, Spidlen V and Mukensnabl P: Diagnostic problems of abdominal desmoid tumors in various locations. Eur J Radiol 62: 180-185, 2007.

4. Sakorafas GH, Nissotakis C and Peros G: Abdominal desmoid tumors. Surg Oncol 16: 131-142, 2007.

5. Efthimiopoulos GA, Chatzifotiou D, Drogouti M and Zafiriou G: Primary asymptomatic desmoid tumor of the mesentery. Am J Case Rep 16: 160-163, 2015.

6. Mangat C, Inoue S, Kader M, Debelenko L and Onwuzurike N: Sudden progressive abdominal pain due to large peritoneal desmoid tumor: A case report with review of literature. J Ped Surg Case Reports 1: 241-243, 2013
7. Koshariya M, Shukla S, Khan Z, Vikas V, Pratap Singh A, Baghel P, Pendro V, Kirti Jain V, Jagdish Jai S, Kumar S and Songra MC: Giant desmoid tumor of the anterior abdominal wall in a young female: A case report. Case Rep Surg 2013: 780862, 2013.

8. Mirza RA, Ghesani MV and Enker WE: Radiological case: Intra-abdominal desmoid tumor. Appl Radiol 43: 20-21, 2014.

9. Chen CB, Chiou YY, Chen $\mathrm{CH}$, Chou YH, Chiang JH and Chang CY. Sonographic and computed tomography findings of intra-abdominal desmoid tumor. J Chin Med Assoc 73: 393-395, 2010.

10. Gari MK, Guraya SY, Hussein AM and Hego MM: Giant mesenteric fibromatosis: Report of a case and review of the literature. World J Gastrointest Surg 4: 79-82, 2012.

11. Shah M and Azam B: Case report of an intra-abdominal desmoid tumour presenting with bowel perforation. Mcgill J Med 10: 90-92, 2007. 